\title{
Y-27632, a Rho-kinase inhibitor, attenuates myocardial ischemia-reperfusion injury in rats
}

\author{
LI-YA DONG ${ }^{1,2}$, XIAO-XIAO QIU ${ }^{3}$, YU ZHUANG $^{4}$ and SONG XUE ${ }^{1}$ \\ ${ }^{1}$ Department of Cardiovascular Surgery, Renji Hospital, School of Medicine, Shanghai Jiao Tong University, \\ Shanghai 200127; ${ }^{2}$ Department of Cardiothoracic Surgery, Xinhua Hospital, Shanghai Jiao Tong University \\ School of Medicine, Shanghai 200092; ${ }^{3}$ Department of Pathophysiology, Wenzhou Medical University, \\ Wenzhou, Zhejiang 325000; ${ }^{4}$ Department of Cardiovascular Surgery, Shanghai General Hospital, \\ Shanghai Jiao Tong University School of Medicine, Shanghai 200080, P.R. China
}

Received October 12, 2018; Accepted February 8, 2019

DOI: $10.3892 /$ ijmm.2019.4097

\begin{abstract}
The present study aimed to evaluate the cardioprotective effects of a Rho-kinase inhibitor, Y-27632, and the underlying mechanisms. A rat model of myocardial ischemia-reperfusion (I/R) injury was generated by ligation of the coronary artery, and global ischemia of isolated rat hearts was conducted using the Langendorff system. Staining with triphenyltetrazolium chloride (TTC) and hematoxylin and eosin was performed to analyze the myocardial infarct size and histopathological alterations of the I/R-induced rat heart. In addition, coronary flow, myocardial contractility and an electrocardiogram were analyzed. The effects of Y-27632 on inflammatory cytokines and cardiac enzymes in the serum were assessed by ELISA. The expression of apoptosis- and inflammation-associated proteins was also analyzed via western blotting. Rats in the Y-27632 group exhibited alleviated myocardial I/R injury according to TTC staining and histopathological diagnosis. Additionally, Y-27632 restored the ST segment. The data of coronary flow and myocardial contractility in isolated rat hearts indicated that Y-27632 improved heart function following I/R. The levels of inflammatory cytokines and cardiac enzymes in the serum were downregulated by Y-27632. The mitogen-activated protein kinase (MAPK) and nuclear factor (NF) $\kappa \mathrm{B}$ signaling pathways were inhibited by Y-27632. Furthermore, apoptosis-associated protein expression in rats and the isolated hearts was effectively inhibited by Y-27632. In conclusion, the findings of the present study indicated that Y-27632 attenuated myocardial injury via inhibiting the activation of the MAPK and $\mathrm{NF}-\kappa \mathrm{B}$ signaling
\end{abstract}

Correspondence to: Professor Song Xue, Department of Cardiovascular Surgery, Renji Hospital, School of Medicine, Shanghai Jiao Tong University, 160 Pujian Road, Shanghai 200127, P.R. China

E-mail: renjixuesong@126.com

Key words: Rho-kinase inhibition, Y-27632, myocardial ischemia-reperfusion, inflammation, apoptosis pathways; thus, apoptosis and the inflammatory response were suppressed.

\section{Introduction}

Cardiovascular diseases are associated with high morbidity and mortality rates worldwide. Restoring the blood supply following myocardial ischemia may alleviate ischemic injury; however, this process can promote damage to the ischemic myocardium, which is identified as ischemia-reperfusion (I/R) injury (1). It has been reported that $I / R$ injury induces damage associated with functional impairment of the heart and arrhythmia (2). Therefore, it is important to alleviate I/R injury when treating ischemic heart disease. In clinical settings, drugs including diltiazem, are mainly used in response to I/R injury. A previous study demonstrated that apoptosis and inflammation are involved in the development of I/R injuries (3), whereas anti-inflammatory and antiapoptotic effects have been reported to be associated with myocardial protection (4).

As a serine/threonine protein kinase of the Rho family, Rho-associated protein kinase (ROCK) acts as a molecular switch of the cellular signaling pathway, and may be activated by RhoA. The RhoA/ROCK signaling pathway has been reported to be involved in numerous diseases, including atherosclerosis, cardiac hypertrophy, heart failure and diabetes-associated diseases. RhoA also serves a central role in signal transduction. It has been suggested that activation of the mitogen-activated protein kinase (MAPK) and nuclear factor $(\mathrm{NF})-\kappa \mathrm{B}$ signaling pathways is regulated by RhoA/ROCK signaling (5). As a selective Rho-kinase inhibitor, (+)-(R)-trans-4-(1-aminoethyl)-N-(4-pyridyl) cyclohexane carboxamide (Y-27632) has been used in numerous experiments (6). Of note, Y-27632 has been observed to exhibit protective effects against I/R cardiac injury in animals (7). Y-27632 has also been reported to suppress the apoptosis of human cardiac stem cells (8). ROCK inhibitors have been demonstrated to protect against I/R injury; however, the underlying molecular mechanism requires further investigation. Therefore, the present study aimed to investigate the pharmacological effects of Y-27632 on I/R-induced heart injury and the potential underlying mechanisms. 


\section{Materials and methods}

Reagents. Y-27632 was purchased from Tocris Cookson, Ltd. (Bristol, UK). All primary antibodies used in the present study, including anti-RhoA, -ROCK1, -c-Jun N-terminal kinase (JNK), -phosphorylated (P)-JNK, -extracellular signal-regulated kinase (ERK), -P-ERK, -P-P38, -P38, -P-NF- $\mathrm{KB},-\mathrm{NF}-\mathrm{\kappa B}$,

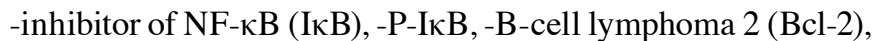
-Bcl-2-associated X (Bax), -Caspase-3, -Caspase-9 and -GAPDH, were obtained from Cell Signaling Technology, Inc. (Danvers, MA, USA). ELISA kits for the detection of interleukin (IL)-6, tumor necrosis factor (TNF)- $\alpha$ and IL-1 $\beta$ were purchased by R\&D Systems, Inc. (Minneapolis, MN, USA). Creatine kinase (CK) and lactate dehydrogenase (LDH) assay kits were obtained from MultiSciences (Lianke) Biotech Co., Ltd. (Hangzhou, China).

Animals. Male Sprague-Dawley rats (8 weeks old; 250-280 g) were obtained from Beijing Vital River Laboratory Animal Technology Co., Ltd. (Beijing, China) and housed together under a 12-h light/dark cycle, with free access to food and water, at a constant temperature of $22-24^{\circ} \mathrm{C}$ and relative humidity of $50 \pm 5 \%$. All animal treatments in the present study were performed humanely and following the institutional and national guidelines for ethical animal research. All experimental procedures were conducted according to the guidelines and following approval from the Ethical Committee of Experimental Animal Care at Wenzhou Medical University (Wenzhou, China).

Myocardial I/R model. The rats were anesthetized via $2 \%$ isoflurane inhalation prior to establishing the I/R rat model by ligation of the left descending coronary artery, as previously described (9). The rats were anesthetized and restrained; the trachea was then incised and wrapped in catgut for later use. An incision was then made in the skin at the position of the heart, and the underlying ribs were exposed by blunt dissection to provide convenient access during the heart procedure. Following exposure of the heart, the left anterior descending coronary artery was blocked with a specific suture. A positive pressure respirator was applied to provide respiratory function for the experimental rats during the procedure. Elevation of the ST segment was used to confirm successful implementation of the surgical model. Recovery of a strong, regular heart rhythm indicated that the thoracic cavity was ready to be sutured. The suture of rats in the sham group was not tied. The rats were randomly divided into four groups: i) Sham; ii) $I / R$; iii) $I / R+$ diltiazem (Dil; $I / R+10 \mathrm{mg} / \mathrm{kg}$ Dil); and iv) $\mathrm{I} / \mathrm{R}+\mathrm{Y}-27632(\mathrm{I} / \mathrm{R}+5 \mathrm{mg} / \mathrm{kg} \mathrm{Y}-27632)$. The rats in the Y-27632 and Dil groups were treated continuously with Y-27632 or Dil for 5 days following I/R. At $24 \mathrm{~h}$ following the final administration of Y-27632 or Dil, the rats were sacrificed by $\mathrm{CO}_{2}$ asphyxiation with $20 \%$ volume displacement $/ \mathrm{min} ; 8-\mathrm{ml}$ blood samples and myocardial tissues were then collected.

Langendorff-perfused heart model. Under anesthesia, the rats were administered with heparin $(250 \mathrm{U} / \mathrm{kg})$ intraperitoneally and the heart was excised following retrograde perfusion with complex Krebs-Henseleit (K-H) buffer at a pressure of $70 \mathrm{~cm}$ $\mathrm{H}_{2} \mathrm{O}$ using the Langendorff system. The heart and perfusate were maintained at $37^{\circ} \mathrm{C}$ with a water jacket. Subsequently, $\mathrm{K}-\mathrm{H}$ buffer was applied for the perfusion of the isolated hearts under $80-90 \mathrm{mmHg}$. The rats were then randomly divided into four groups: i) Control, hearts were perfused for a stabilization period of $80 \mathrm{~min}$; ii) I/R, hearts were equilibrated for $20 \mathrm{~min}$ followed by global ischemia for $30 \mathrm{~min}$. K-H buffer was used for reperfusion for $30 \mathrm{~min}$; iii) $\mathrm{I} / \mathrm{R}+\mathrm{Dil}$; and iv) $\mathrm{I} / \mathrm{R}+\mathrm{Y}-27632$, hearts were processed according to the method of the $I / R$ group; however, reperfusion was conducted using Y-27632 $(0.5 \mathrm{mg} / \mathrm{kg})$ or Dil $(0.2 \mathrm{mg} / \mathrm{kg})$ dissolved K-H buffer for $30 \mathrm{~min}$.

Coronary flow and myocardial contractility analysis. Following reperfusion for various durations, the K-H effluent flow was measured as the coronary flow; a flow rate of $>8 \mathrm{ml} / \mathrm{min}$ during the stabilization period was selected. Coronary effluent was collected following downstream analysis. A portable heart clip was used to record the contractile force of the myocardium during the experiment.

Electrocardiogram (ECG). In the present study, an ECG was generated using needle electrodes and the BL-420 S biological function experiment system (Chengdu TME Technology, Co., Ltd., Chengdu, China). ST segment elevation on the ECG was considered to be an indicator of ischemia.

Assessment of myocardial infarct area. Triphenyltetrazolium (TTC) staining (Sigma-Aldrich; Merck KGaA, Darmstadt, Germany) was conducted to determine the myocardial infarct size. Briefly, the hearts were incubated at $-20^{\circ} \mathrm{C}$ for $15 \mathrm{~min}$. The hearts were cut into five parallel pieces and placed in $1 \%$ TTC for $15 \mathrm{~min}$ at $37^{\circ} \mathrm{C}$ in the dark. The myocardial infarct ratio [(infarct area/whole heart area) $\mathrm{x}$ percentage] was calculated using image analysis software (Image-Pro Plus version 4.1; Media Cybernetics, Inc., Rockville, MD, USA).

Determination of inflammatory cytokines in the serum. The levels of inflammatory markers, including IL-6, IL- $1 \beta$ and TNF- $\alpha$, in the serum were examined using mice ELISA kits according to the manufacturer's protocols.

Analysis of cardiac enzymes. The serum levels of CK and LDH were measured using the commercial kits according to the manufacturer's protocols.

Histological evaluation. The morphology of the myocardial tissues was examined using hematoxylin and eosin (H\&E) staining. Following dehydration with 80,90 and $100 \%$ ethanol and n-butanol, the myocardial tissue was waxed in a $60^{\circ} \mathrm{C}$ wax box and then embedded in paraffin. Tissue sections $(5-\mu \mathrm{m})$ were dried at $45^{\circ} \mathrm{C}$ and obtained from each paraffin block. The sections were heated at $60^{\circ} \mathrm{C}$ for $1 \mathrm{~h}$ and dewaxed with xylene. Following hydration, the sections were stained with $\mathrm{H} \& \mathrm{E}$, dehydrated with gradient ethanol, cleared with xylene and mounted with neutral gum. Images were captured using a light microscope (BX41; Olympus Corporation, Tokyo, Japan).

Western blotting. Total protein was extracted from heart samples and analyzed by western blotting with the specific antibodies described. Briefly, the samples were collected and 


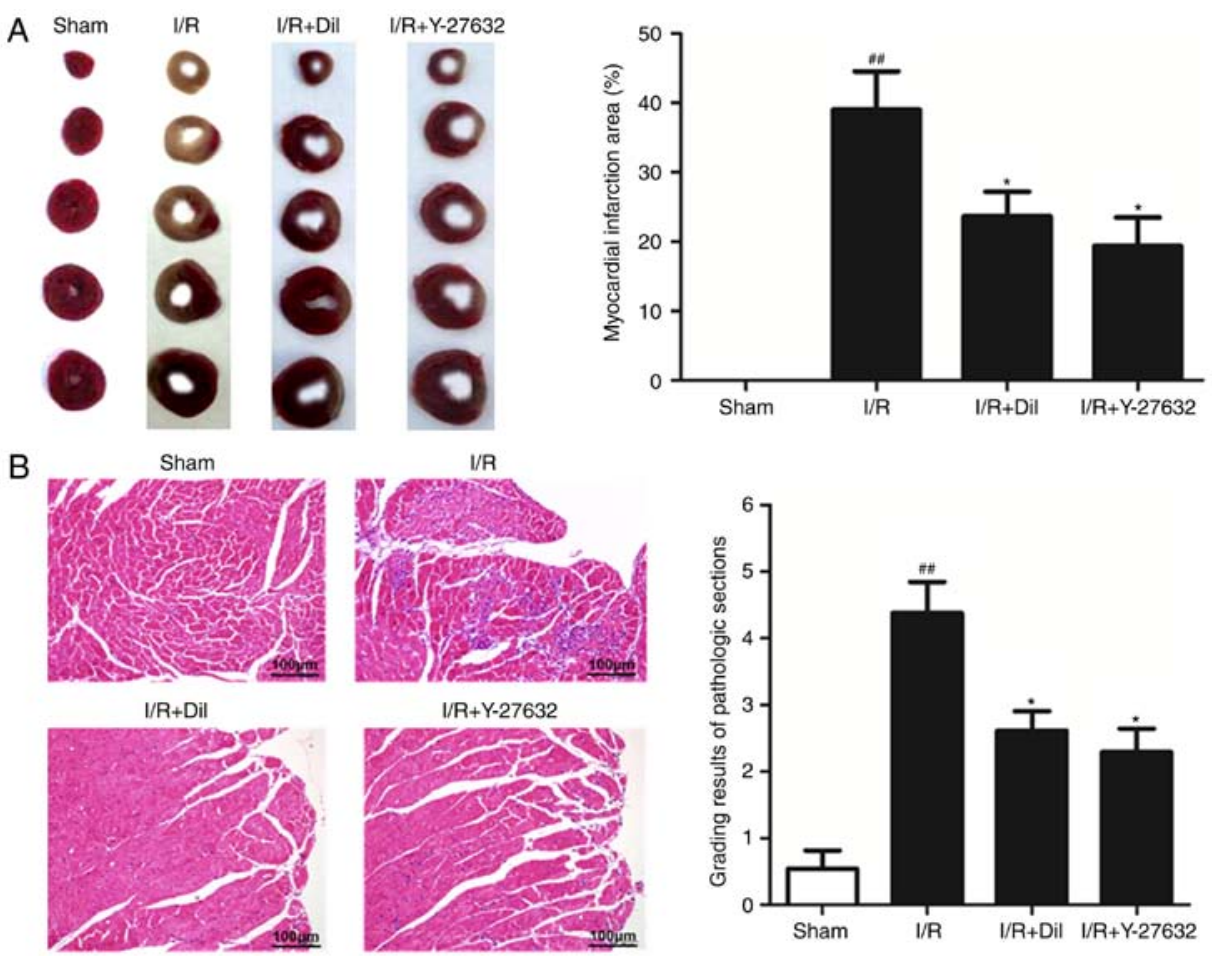

Figure 1. Effects of Y-27632 on myocardial infarct size and histology. Effect of Y-27632 on (A) myocardial infarct size and (B) myocardial histology in $\mathrm{I} / \mathrm{R}$-induced isolated heart. Original magnification, $\mathrm{x} 200$. The data are expressed as the mean \pm standard deviation. ${ }^{\# \#} \mathrm{P}<0.01 \mathrm{vs}$. sham group; ${ }^{*} \mathrm{P}<0.05$ vs. I/R group. I/R, ischemia-reperfusion; Dil, diltiazem.

homogenized, and then lysed in extraction buffer for $1 \mathrm{~h}$ on ice. The total protein content was quantified using a Bicinchoninic Acid protein assay kit (Beyotime Institute of Biotechnology, Nanjing, China). Equal quantities of protein $(20 \mu \mathrm{g})$ were separated by $8-12 \%$ SDS-PAGE and then electrotransferred onto polyvinylidene difluoride (PVDF) membranes, which were blocked with skim milk at room temperature for $2 \mathrm{~h}$. Following blocking, the PVDF membranes were incubated with specific antibodies against RhoA (1:1,000; cat. no. 2117), ROCK1 (1:1,000; cat. no. 4035), P-JNK (1:1,000; cat. no. 9255), JNK (1:1,000; cat. no. 9252, P-ERK (1:1,000; cat. no. 8544), ERK (1:1,000; cat. no. 4348), P-P38 (1:1,000; cat. no. 4511), P38 (1:1,000; cat. no. 8690), P-NF-кB (1:1,000; cat. no. 3033),

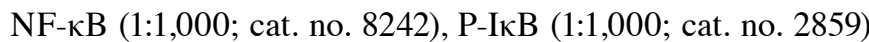
IкB (1:1,000; cat. no. 4812), Bax (1:1,000; cat. no. 2772), Bcl-2 (1:1,000; cat. no. 2764), Caspase-3 (1:1,000; cat. no. 9662), Caspase-9 (1:1,000; cat. no. 9508) and GAPDH (1:2,000; cat. no. 5174; all from Cell Signaling Technology, Inc.) overnight at $4^{\circ} \mathrm{C}$. On the second day, the PVDF membranes were incubated with a secondary antibody [horseradish peroxidase-labeled mouse anti-rabbit immunoglobulin (Ig)-G (1:5,000; cat. no. 5127; Cell Signaling Technology, Inc.); and horseradish peroxidase-labeled rabbit anti-mouse IgG (1:5,000; cat. no. 58802; Cell Signaling Technology, Inc.)], at room temperature for $1 \mathrm{~h}$ following three washes with tris-buffered saline with Tween-20. Subsequently, the immunoreactive bands were visualized with a gel imaging system (Tanon 5200; Tanon Science and Technology Co., Ltd., Shanghai, China).

Statistical analysis. The data are presented as the mean \pm standard deviation, and were analyzed by one-way analysis of variance followed by Tukey's post hoc test using
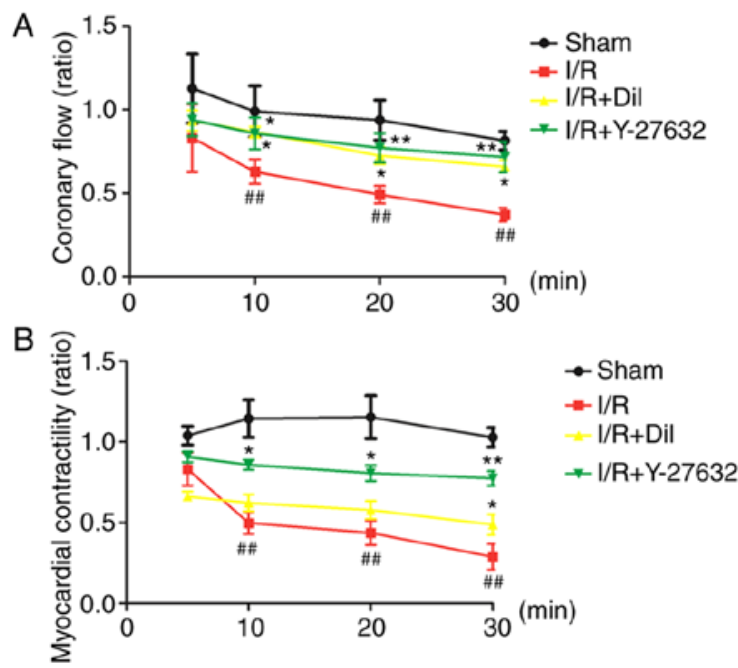

Figure 2. Effect of Y-27632 on coronary flow and myocardial contractility. Effect of Y-27632 on (A) coronary flow and (B) myocardial contractility in the I/R-induced isolated heart. The data are expressed as the mean \pm standard deviation. ${ }^{\# \#} \mathrm{P}<0.01$ vs. sham group; ${ }^{*} \mathrm{P}<0.05$ and ${ }^{* *} \mathrm{P}<0.01$ vs. I/R group. $\mathrm{I} / \mathrm{R}$, ischemia-reperfusion; Dil, diltiazem.

GraphPad Prism software 6.0 (GraphPad Software, Inc., La Jolla, CA, USA). P $<0.05$ was considered to indicate a statistically significant difference.

\section{Results}

Effects of Y-27632 on myocardial infarct size. To determine whether Y-27632 exhibits myocardial protective effects, the infarct size of rat hearts was analyzed by TTC staining. As 

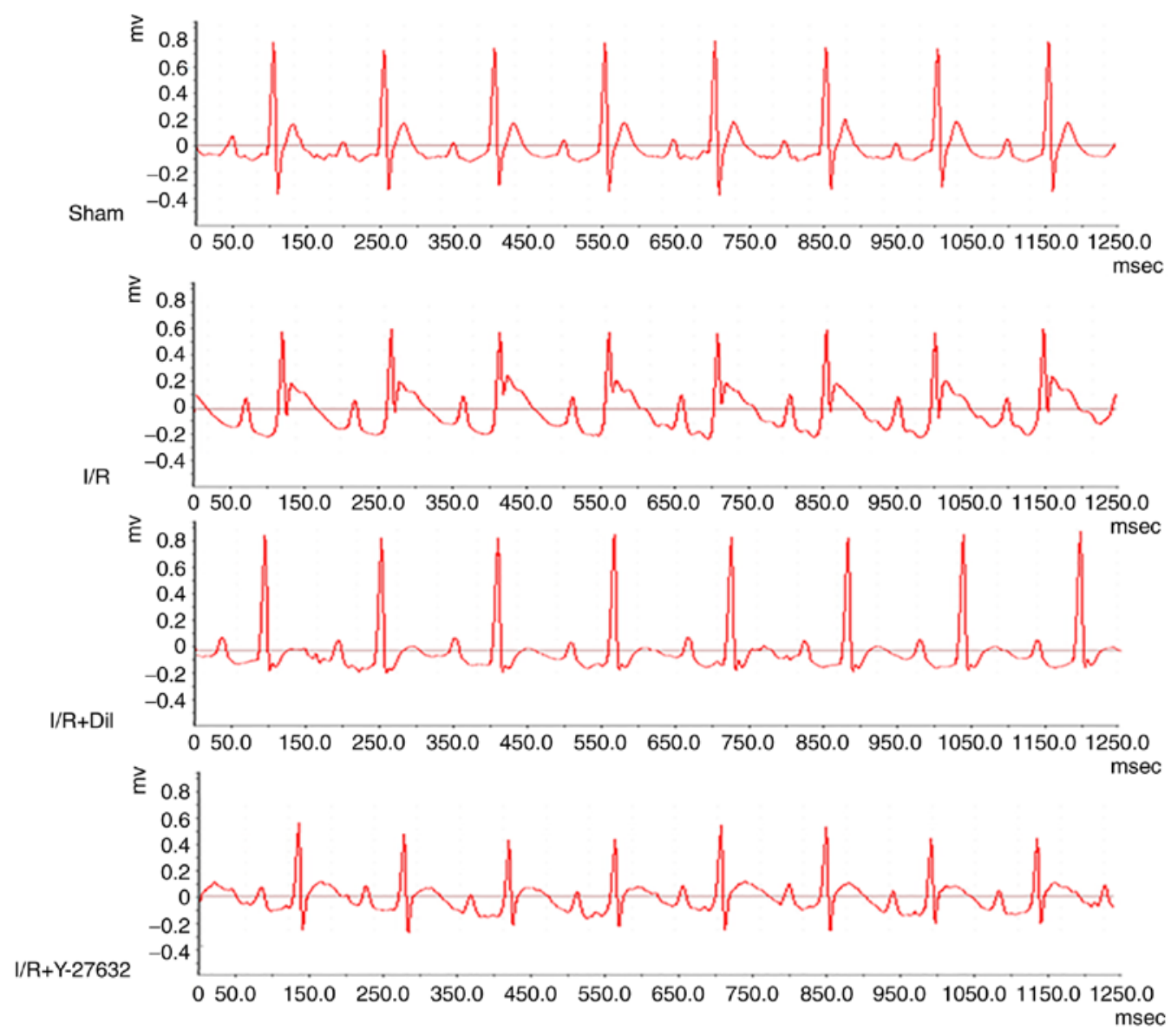

Figure 3. Effects of Y-27632 on ST-segment elevation. I/R, ischemia-reperfusion; Dil, diltiazem.

presented in Fig. 1A, compared with the sham group, I/R markedly increased the infarct size, which was significantly reduced following treatment with Y-27632 or Dil.

Effects of $Y-27632$ on myocardial histology. To investigate the effects of Y-27632 on myocardial injuries, histopathological alterations of the myocardial tissues were analyzed. As presented in Fig. 1B, extensive inflammatory cells and the notable destruction of myocardial cellular structure were observed in the I/R group compared with the sham group. Additionally, treatment with Y-27632 or Dil markedly attenuated the histological alterations induced by I/R.

Effects of Y-27632 on coronary flow in the $I / R$-induced isolated heart. As presented in Fig. 2A, the I/R group exhibited significant decreases in the ratio of coronary flow compared with that in the sham group $(\mathrm{P}<0.01)$. By contrast, treatment with Y-27632 or Dil led to notable increases in the ratio of coronary flow.

Effects of Y-27632 on myocardial contractility in the $I / R$-induced isolated heart. Analysis of the ratio of myocardial contractility of isolated rat hearts revealed that the model group exhibited significantly a reduced ratio of contractility following the inhibition of K-H flow compared with that in the sham group $(\mathrm{P}<0.01)$. By contrast, treatment with $\mathrm{Y}-27632$ or Dil led to a notable increase in the myocardial contractility (Fig. 2B).
Effects of Y-27632 on ST-segment elevation. As presented in Fig. 3, an increase in ST-segment and a decrease in R-amplitude were observed in the I/R group compared with the sham group; however, these effects were reversed following treatment with Y-27632 or Dil.

Effects of Y-27632 on inflammatory cytokines in the serum. To investigate the potential anti-inflammatory effects of Y-27632, the serum levels of IL- 6 , TNF- $\alpha$ and IL- $1 \beta$ were measured by ELISA. As presented in Fig. 4A, the levels of IL-6, TNF- $\alpha$ and IL-1 $\beta$ were significantly upregulated in the I/R model group compared with those in the sham group. Treatment with Y-27632 or Dil significantly reduced the levels of inflammatory cytokines compared with those in the I/R group.

Effects of Y-27632 on cardiac enzymes. As representative indicators of myocardial injury, the levels of CK and LDH in the serum were analyzed. As presented in Fig. 4B, the serum levels of CK and LDH were significantly upregulated in the $\mathrm{I} / \mathrm{R}$ group compared with the those in the sham group $(\mathrm{P}<0.01$, respectively). By contrast, treatment with Y-27632 or Dil effectively suppressed the levels of CK and LDH.

Effects of $Y-27632$ on the RhoA/ROCK and MAPK/NF- $\kappa B$ signaling pathways, and apoptosis in the $I / R$-induced heart. To determine the intracellular signaling pathway underlying the myocardial protective effects of $\mathrm{Y}-27632$ in I/R, the RhoA/ROCK and MAPK/NF- $\mathrm{KB}$ signaling pathways, and 

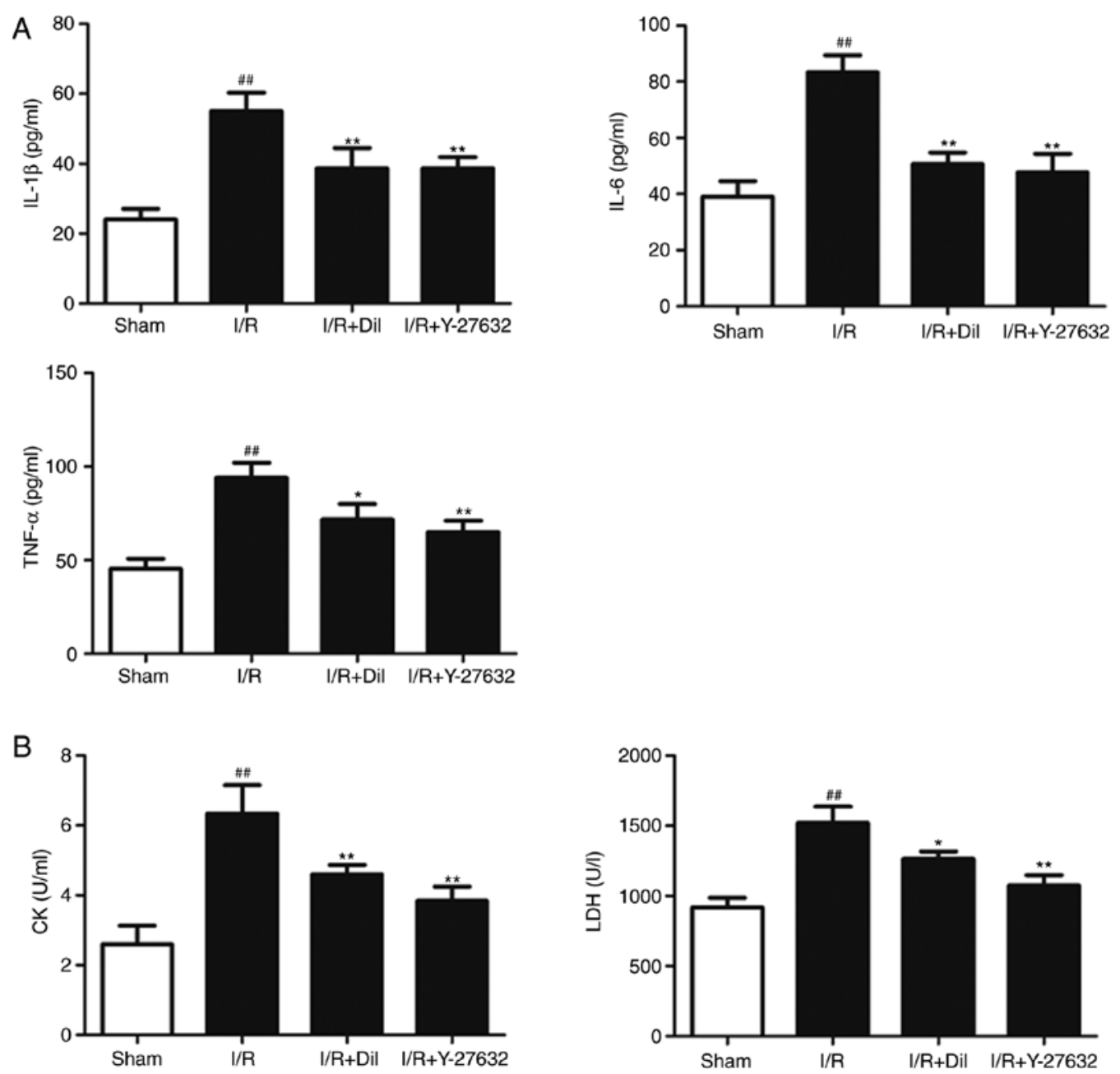

Figure 4. Effects of Y-27632 on inflammatory cytokines and cardiac markers. Effects of Y-27632 on (A) inflammatory cytokines and (B) cardiac marker enzymes in serum. The data are expressed as the mean \pm standard deviation. ${ }^{\# \#} \mathrm{P}<0.01$ vs. control group; ${ }^{*} \mathrm{P}<0.05$ and ${ }^{* * *} \mathrm{P}<0.01$ vs. I/R group. I/R, ischemia-reperfusion; Dil, diltiazem; IL, interleukin; TNF- $\alpha$, tumor necrosis factor- $\alpha$; CK, creatine kinase; $\mathrm{LDH}$, lactate dehydrogenase.
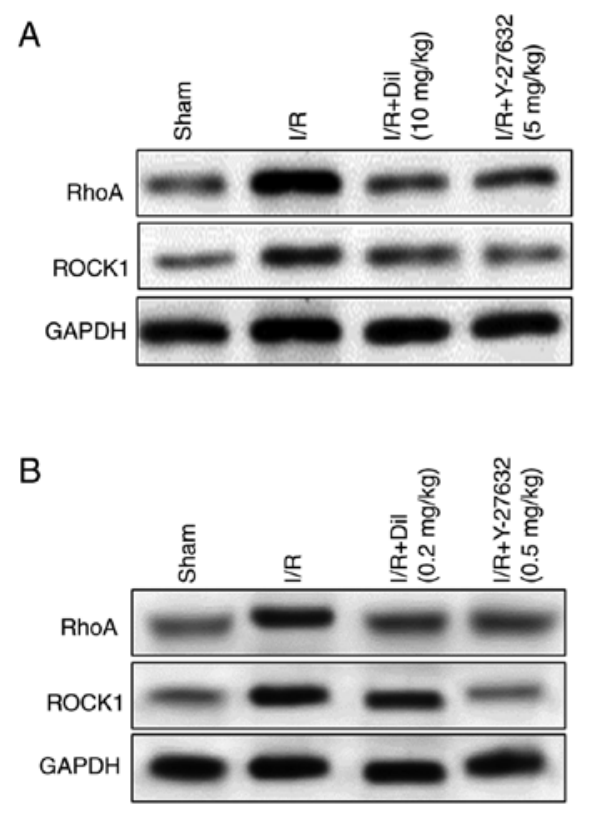
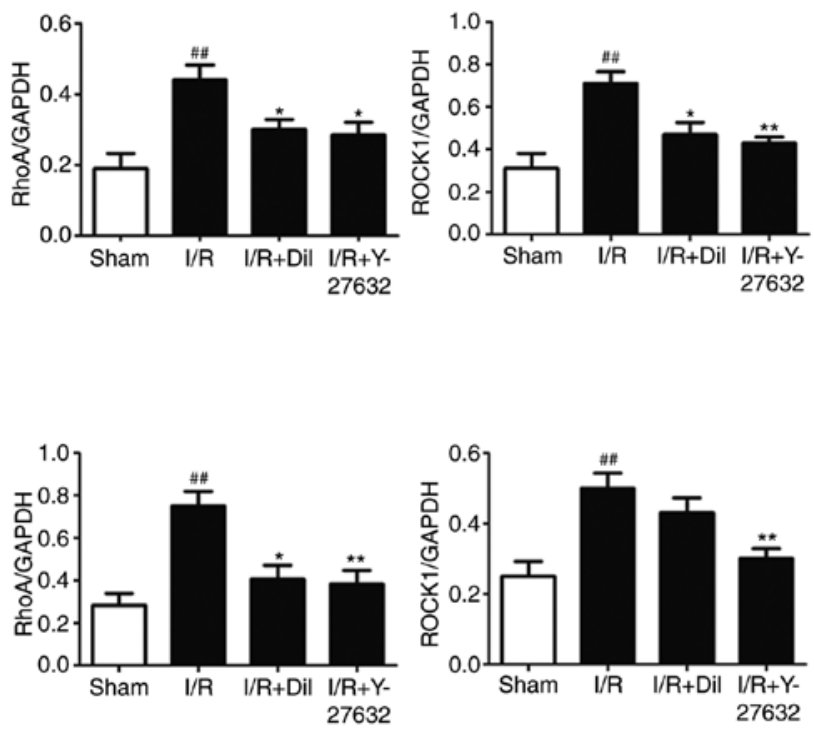

Figure 5.Effects of Y-27632 on the RhoA/ROCK pathway.RhoA/ROCK pathway in the hearts of (A) left coronary artery ligation rats and (B) Langendorff-perfused rats. The data are expressed as the mean \pm standard deviation. ${ }^{\# \#} \mathrm{P}<0.01$ vs. sham/control group; ${ }^{*} \mathrm{P}<0.05$ and ${ }^{* * *} \mathrm{P}<0.01$ vs. I/R group. I/R, ischemia-reperfusion; Dil, diltiazem; ROCK, Rho-associated protein kinase. 

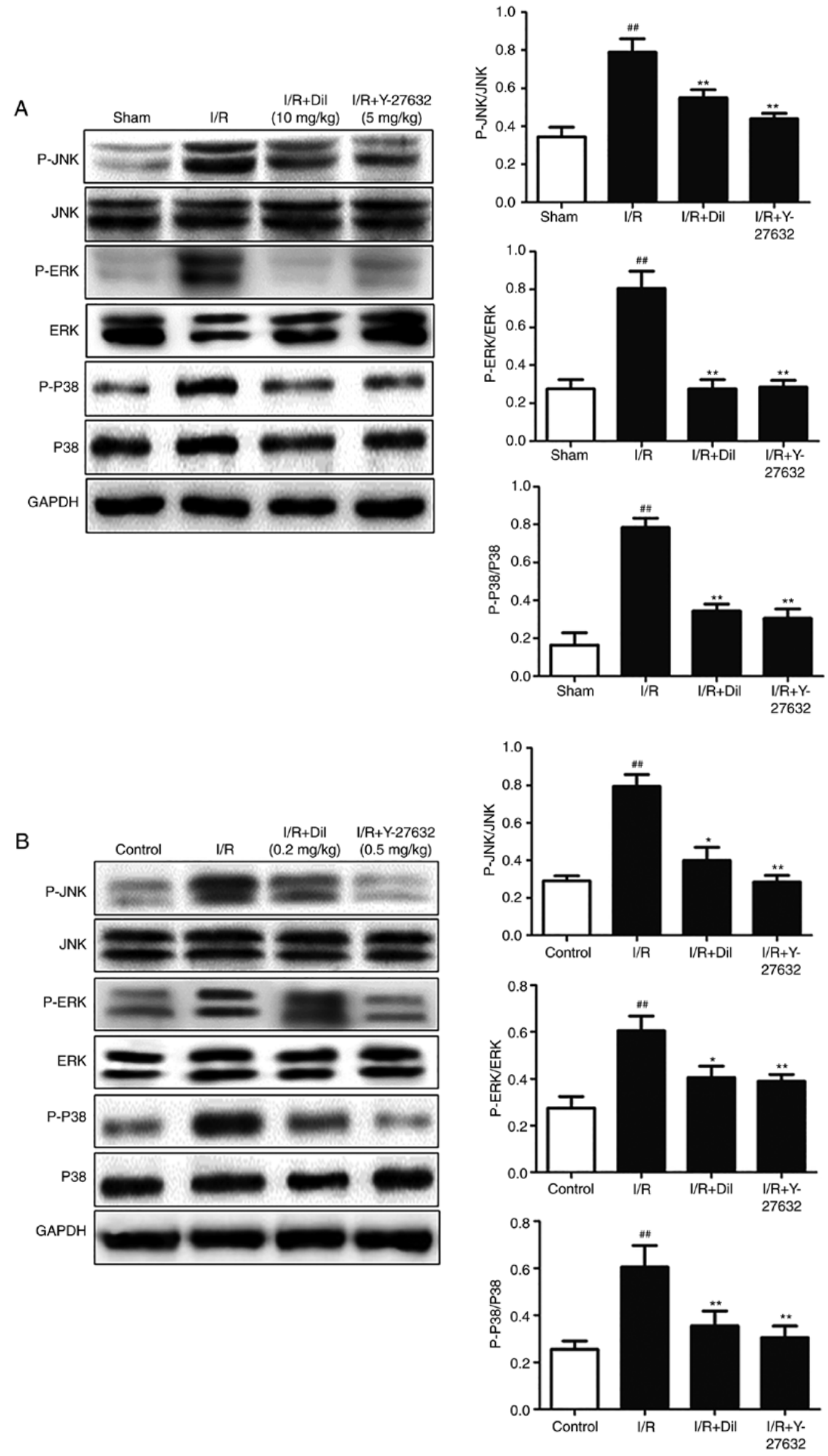

Figure 6. Effects of Y-27632 on the phosphorylation of mitogen-activated protein kinases. Effects in the hearts of (A) left coronary artery ligation rats and (B) Langendorff-perfused rats hearts. The data are expressed as the mean \pm standard deviation. ${ }^{\# \#} \mathrm{P}<0.01 \mathrm{vs}$. sham $/$ control group; ${ }^{*} \mathrm{P}<0.05$ and ${ }^{* *} \mathrm{P}<0.01 \mathrm{vs}$. I/R group. I/R, ischemia-reperfusion; Dil, diltiazem; JNK, c-Jun N-terminal kinase; ERK, extracellular signal-regulated kinase; P-, phosphorylated. 
A
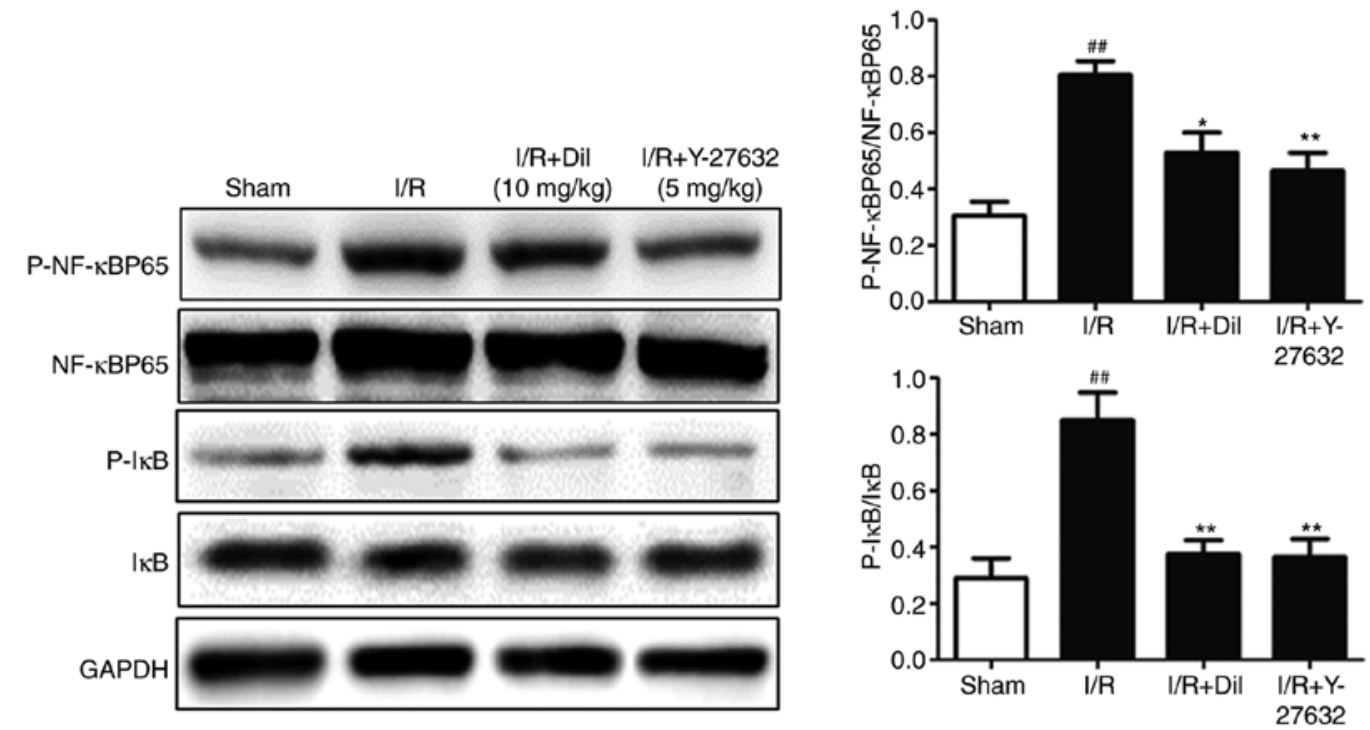

B
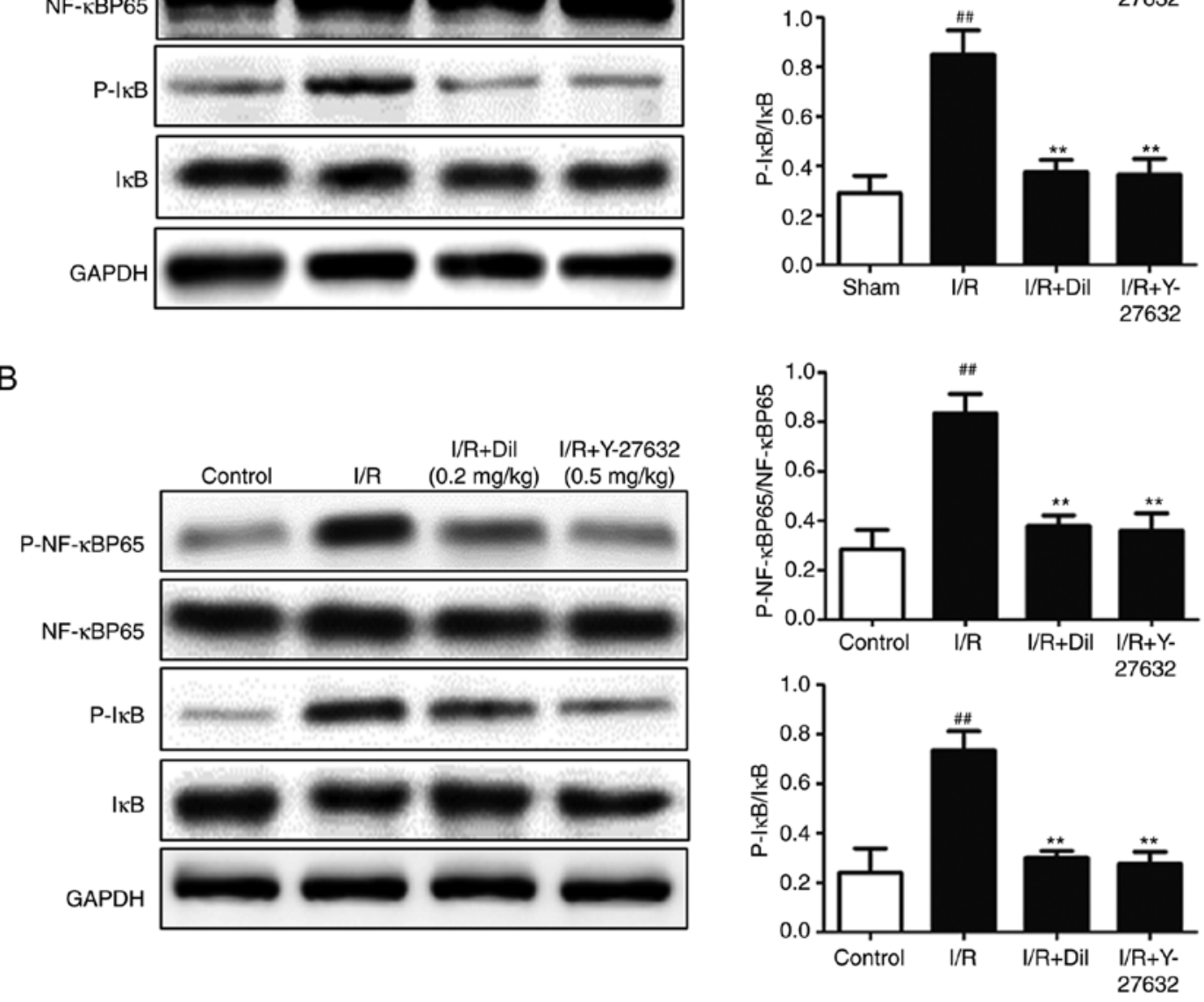

Figure 7. Effects of Y-27632 on the activation of NF-кB. Hearts of (A) left coronary artery ligation rats and (B) Langendorff-perfused rats. The data are expressed as the mean \pm standard deviation. ${ }^{\# \#} \mathrm{P}<0.01$ vs. sham/control group; ${ }^{*} \mathrm{P}<0.05$ and ${ }^{* *} \mathrm{P}<0.01$ vs. I/R group. I/R, ischemia-reperfusion; Dil, diltiazem;

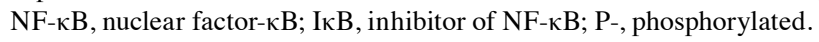

apoptosis were investigated. Upregulated expression levels of RhoA and ROCK1 (Fig. 5A and B) and increased phosphorylation of ERK1/2, JNK and P38 proteins (Fig. 6A and B) were observed in the I/R group; however, treatment with Y-27632 or Dil treatment notably reversed the effects of $I / R$ on the expression of the aforementioned proteins.

To further investigate the downstream mechanism underlying the effects of Y-27632 on the I/R-induced heart in rats, the expression levels of P-IкB and P-NF- $\kappa \mathrm{B}$ were determined (Fig. 7A and B). Western blot analysis demonstrated

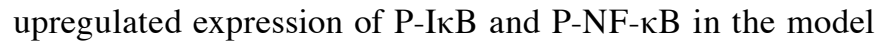
group compared with the sham group. Following treatment with Y-27632 or Dil, significantly suppressed expression levels

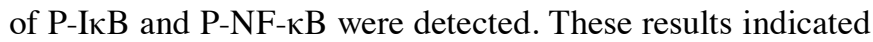
that the inhibition of ROCK1 may prevent the activation of NF-кB.

As presented in Fig. 8A and B, the expression levels of apoptosis-associated proteins were detected to investigate the mechanism underlying the effect of Y-27632 on cardiomyocyte apoptosis. I/R injury markedly increased the expression of Bax, Caspase-3 and Caspase-9, which was associated with reduced expression levels of Bcl-2. Treatment with Y-27632 or Dil markedly reversed the effects of I/R injury on the expression of the aforementioned proteins. These results indicated that the inhibition of ROCK1 may suppress apoptosis.

\section{Discussion}

At present, the prevalence and mortality rates of cardiovascular diseases have significantly increased with the aging of populations and alterations in lifestyle (10). Cardiovascular diseases are the main cause of mortality in the Chinese population and account for $>40 \%$ of the reported mortality rate, which may increase in the next 10 years (11). The therapeutic approaches and agents available have markedly improved; however, the treatment of myocardial ischemia 
A

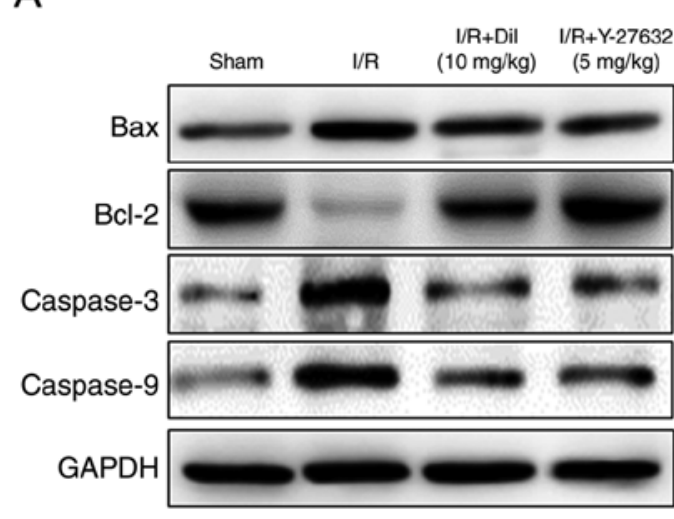

B

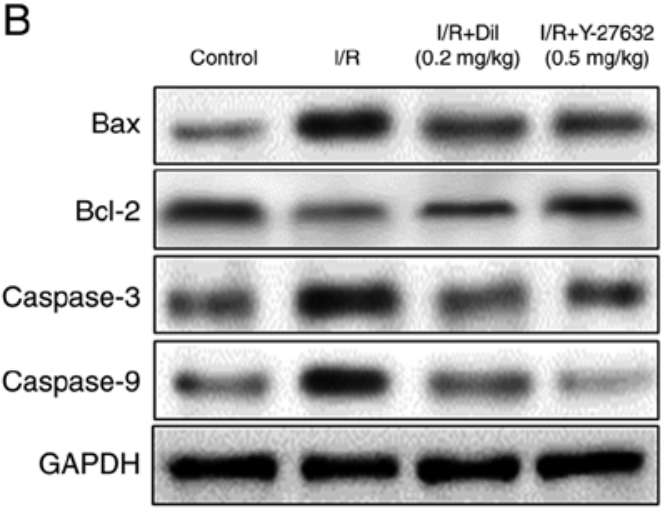

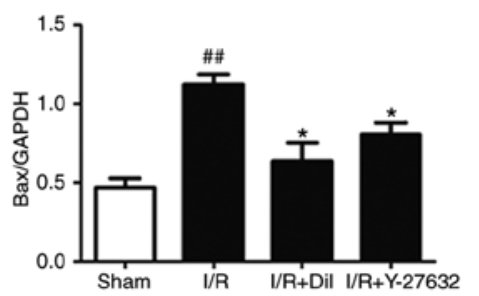
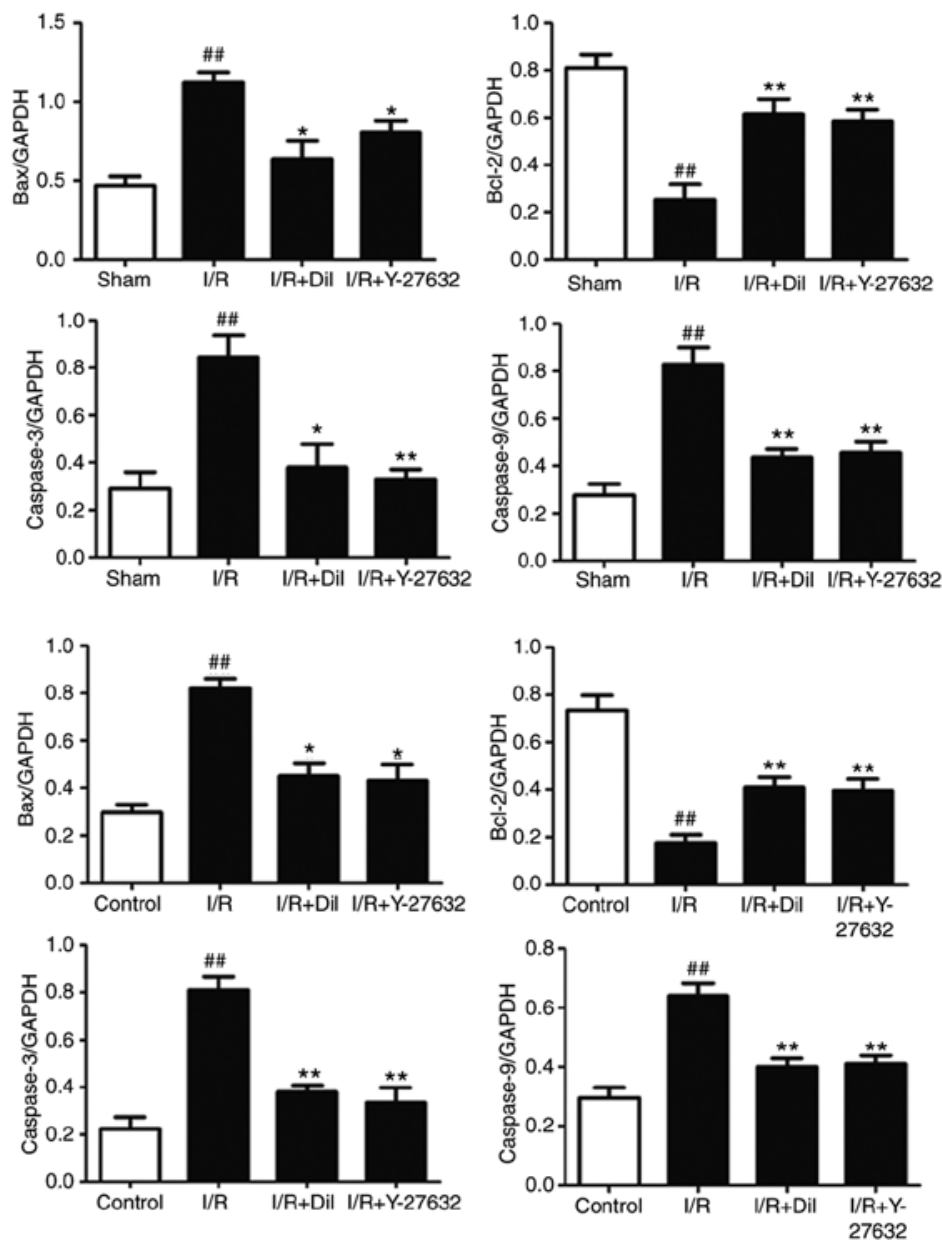

Figure 8. Effect of Y-27632 on apoptosis. Apoptosis-associated protein expression in the hearts of (A) left coronary artery ligation rats and (B) Langendorff-perfused rats. The data are expressed as the mean \pm standard deviation. ${ }^{\# /} \mathrm{P}<0.01$ vs. sham $/$ control group; ${ }^{*} \mathrm{P}<0.05$ and ${ }^{* *} \mathrm{P}<0.01 \mathrm{vs}$. I/R group. I/R, ischemia-reperfusion; Dil, diltiazem; Bcl-2, B-cell lymphoma 2; Bax, Bcl-2-associated X.

remains ineffective. Therefore, further investigation into treating myocardial ischemia is required. In the present study, the cardioprotective effects of Y-27632 were determined via TTC and H\&E staining, and analyzing ST-segment elevations. These observations were supported by analyzing coronary flow and myocardial contractility.

The Rho/ROCK signaling pathway has been associated with the growth, proliferation and differentiation of cells, which are widely involved in various pathophysiological processes. Rho A is a small GTP-binding protein, whereas ROCK is the downstream effector protein of Rho A (12). ROCK is a serine/threonine protein kinase involved in the regulation of numerous cellular functions, including migration, contraction and adhesion. Blood pressure, cardiac hypertrophy, heart failure and other cardiovascular diseases have been associated with the activity of ROCK (13). As the downstream molecule of ROCK, $\mathrm{NF}-\mathrm{kB}$ is a nuclear transcription factor with multi-directional regulation, and regulates the expression of genes associated with immune, stress and inflammatory responses. It has been reported that $\mathrm{I} / \mathrm{R}$ of the heart can induce the overexpression of inflammatory and apoptotic cytokines (14-16). Increasing evidence has indicated that the Rho/ROCK/NF- $\mathrm{KB}$ signaling pathway serves important roles in cardiomyocytes and experimental myocardial infarction (17-19). In the present study, it was determined that Y-27632 successfully inhibited the
Rho/ROCK signaling pathway and may lead to suppression of the activation of NF- $\kappa \mathrm{B}$.

MAPKs, including P38 MAPK, JNK and ERK, serve crucial roles in cell signaling transduction (17). Upon stimulation by a variety of stimuli, MAPKs can regulate the growth, proliferation, differentiation and survival of cells. As widely known stress-activated protein kinases, JNK and P38 MAPK promote cellular survival or apoptosis (18). It has been suggested that JNKs induce apoptosis caused by I/R, inflammation or oxidative stress (19). Sustained activation of ERK1/2 in I/R has been reported previously. Furthermore, ROCK has been determined to be associated with the activation of MAPK in cardiovascular diseases. The findings of the present study suggested that the inhibition of ROCK effectively suppressed the phosphorylation of MAPKs.

Myocardial I/R can induce apoptosis in heart tissues. Myocardial infarction has been reported to increase cell permeability, promote the caspase cascade and lead to the apoptosis of cardiomyocytes (20). The harmful stimulus of myocardial infarction promotes Caspase-9 via an apoptotic signaling pathway and induces Caspase-3, a common downstream effector involved in the apoptotic signaling pathway and with a central role in this process. In addition, Bax and Bcl-2 of the Bcl-2 family are the most important regulatory genes in apoptosis. Numerous studies have suggested that Bcl-2, Bax, Caspase-3 
and Caspase-9 are involved in the pathogenesis of myocardial I/R injury. The results of the present study revealed that Y-27632 markedly prevented I/R-induced apoptosis in the rat heart.

In conclusion, the present study demonstrated that Y-27632, a ROCK inhibitor, markedly ameliorated myocardial I/R-induced injury. The protective effects may be due to inhibited MAPK/NF- $\mathrm{BB}$ signaling activation; thus, apoptosis and the inflammatory response are suppressed. The findings of the present study may provide novel insight into potential treatments for cardiovascular diseases; however, further investigation is required.

\section{Acknowledgements}

Not applicable.

\section{Funding}

The present study was supported by the National Natural Science Foundation of China (grant no. 81670225) and Zhejiang Public Welfare Technology Application Research (grant no. 2013C33168).

\section{Availability of data and materials}

The datasets used and/or analyzed during the current study are available from the corresponding author on reasonable request.

\section{Authors' contributions}

LYD, XXQ, YZ and SX contributed to the conception and design of the study, carried out the animal experiments and revised the manuscript. LYD, YZ and SX contributed to the acquisition, analysis and interpretation of the data. LYD and SX revised the manuscript. All authors read and approved the final manuscript.

\section{Ethics approval and consent to participate}

All experimental procedures were approved by the Ethical Committee of Experimental Animal Care at Wenzhou Medical University (Wenzhou, China).

\section{Patient consent for publication}

Not applicable.

\section{Competing interests}

The authors declare that they have no competing interests.

\section{References}

1. Hu Q, Wei B, Wei L, Hua K, Yu X, Li H and Ji H: Sodium tanshinone IIA sulfonate ameliorates ischemia-induced myocardial inflammation and lipid accumulation in Beagle dogs through NLRP3 inflammasome. Int J Cardiol 196: 183-192, 2015.
2. Zhu L, Wei T, Gao J, Chang X, He H, Luo F, Zhou R, Ma C, Liu Y and Yan T: The cardioprotective effect of salidroside against myocardial ischemia reperfusion injury in rats by inhibiting apoptosis and inflammation. Apoptosis 20: 1433-1443, 2015.

3. Weinreuter M, Kreusser MM, Beckendorf J, Schreiter FC, Leuschner F, Lehmann LH, Hofmann KP, Rostosky JS, Diemert N, Xu C, et al: CaM Kinase II mediates maladaptive post-infarct remodeling and pro-inflammatory chemoattractant signaling but not acute myocardial ischemia/reperfusion injury. EMBO Mol Med 6: 1231-1245, 2014.

4. Hua K, Sheng X, Li T, Wang LN, Zhang YH, Huang ZJ and $\mathrm{Ji} \mathrm{H}$ : The edaravone and 3- $n$-butylphthalide ring-opening derivative $10 \mathrm{~b}$ effectively attenuates cerebral ischemia injury in rats. Acta Pharmacol Sin 36: 917-927, 2015.

5. Fierro C, Novoa U, González V, Ocaranza MP and Jalil JE: Simultaneous Rho kinase inhibition in circulating leukocytes and in cardiovascular tissue in rats with high angiotensin converting enzyme levels. Int J Cardiol 215: 309-317, 2016.

6. Xue J, Fan B, Xing Q and Li T: Effect of Y-27632 Rho-kinase inhibitor on proliferation and migration of human glioblastoma cell. J Henan Univ Sci Technol 28: 247-252, 2010.

7. Ikeda F, Terajima H, Shimahara Y, Kondo T and Yamaoka Y: Reduction of hepatic ischemia/reperfusion-induced injury by a specific ROCK/Rho kinase inhibitor Y-27632. J Surg Res 109: $155-160,2003$.

8. Kan L, Smith A, Chen M, Ledford BT, Fan H, Liu Z and He JQ: Rho-associated kinase inhibitor (Y-27632) attenuates doxorubicin-induced apoptosis of human cardiac stem cells. PLoS One 10: e0144513, 2015.

9. Dong LY, Chen F, Xu M, Yao LP, Zhang YJ and Zhuang Y: Quercetin attenuates myocardial ischemia-reperfusion injury via downregulation of the HMGB1-TLR4-NF-kappaB signaling pathway. Am J Transl Res 10: 1273-1283, 2018.

10. Bastien M, Poirier P, Lemieux I and Després JP: Overview of epidemiology and contribution of obesity to cardiovascular disease. Prog Cardiovasc Dis 56: 369-381, 2014.

11. Gu D, Gupta A, Muntner P, Hu S, Duan X, Chen J, Reynolds RF, Whelton PK and He J: Prevalence of cardiovascular disease risk factor clustering among the adult population of China: Results from the International Collaborative Study of Cardiovascular Disease in Asia. Circulation 112: 658-665, 2005.

12. Chen T, Guo Q, Wang H, Zhang H, Wang C, Zhang P, Meng S, Li Y, Ji H and Yan T: Effects of esculetin on lipopolysaccharide (LPS)-induced acute lung injury via regulation of RhoA/Rho kinase/NF-small ka, CyrillicB pathways in vivo and in vitro. Free Radic Res 49: 1459-1468, 2015.

13. Ohki S, Iizuka K, Ishikawa S, Kano M, Dobashi K, Yoshii A, Shimizu Y, Mori M and Morishita Y: A highly selective inhibitor of Rho-associated coiled-coil forming protein kinase, Y-27632, prolongs cardiac allograft survival of the BALB/c-to- $\mathrm{C} 3 \mathrm{H} / \mathrm{He}$ mouse model. J Heart Lung Transplantat 20: 956-963, 2001.

14. Li C, Gao Y, Tian J, Shen J, Xing Y and Liu Z: Sophocarpine administration preserves myocardial function from ischemiareperfusion in rats via NF- $\kappa \mathrm{B}$ inactivation. J Ethnopharmacol 135: 620-625, 2011.

15. Lin J, Wang H, Li J, Wang Q, Zhang S, Feng N, Fan R and Pei J: $\kappa-O p i o i d ~ r e c e p t o r$ stimulation modulates TLR4/NF- $\kappa \mathrm{B}$ signaling in the rat heart subjected to ischemia-reperfusion. Cytokine 61: 842-848, 2013.

16. Henkel T, Machleidt T, Alkalay I, Krönke M, Benneriah Y and Baeuerle PA: Rapid proteolysis of I $\mathrm{KB}-\alpha$ is necessary for activation of transcription factor NF-кB. Nature 365: 182-185, 1993.

17. Johnson GL and Lapadat R: Mitogen-activated protein kinase pathways mediated by ERK, JNK, and p38 protein kinases. Science 298: 1911-1912, 2002.

18. Gaestel M: MAPK-activated protein kinases (MKs): Novel insights and challenges. Front Cell Dev Biol 3: 88, 2016.

19. Iliodromitis EK, Gaitanaki C, Lazou A, Bofilis E, Karavolias GK, Beis I and Kremastinos DT: Dissociation of stress-activated protein kinase (p38-MAPK and JNKs) phosphorylation from the protective effect of preconditioning in vivo. J Mol Cellular Cardiol 34: 1019-1028, 2002.

20. Wei Y, Xu M, Ren Y, Lu G, Xu Y, Song Y and Ji H: The cardioprotection of dihydrotanshinone I against myocardial ischemia-reperfusion injury via inhibition of arachidonic acid w-hydroxylase. Can J Physiol Pharmacol 94: 1267-1275, 2016. 